\title{
Hydroxyethyl starch versus other fluids for non-septic patients in the intensive care unit: a meta-analysis of randomized controlled trials
}

\author{
Bin $\mathrm{He}^{1+}$, Bo Xu ${ }^{1+}$, Xiaoxing $\mathrm{Xu}^{2}$, Lixia $\mathrm{Li}^{3}$, Rongrong Ren ${ }^{1}$, Zhiyu Chen ${ }^{1}$, Jian Xiao ${ }^{4}$, Yingwei Wang ${ }^{{ }^{*}}$ and Bin $\mathrm{Xu}^{5^{*}}$
}

\begin{abstract}
Introduction: Use of hydroxyethyl starch (HES) in septic patients is reported to increase the mortality and incidence of renal replacement therapy (RRT). However, whether or not use of HES would induce the same result in non-septic patients in the intensive care unit (ICU) remains unclear. The objective of this meta-analysis was to evaluate $6 \%$ HES versus other fluids for non-septic ICU patients.
\end{abstract}

Methods: Randomized controlled trials (RCTs) were searched from Pubmed, OvidSP, Embase database and Cochrane Library, published before November, 2013. A meta-analysis was made on the effect of $6 \%$ HES versus other fluids for non-septic ICU patients, including mortality, RRT incidence, bleeding volume, red blood cell (RBC) transfusion and fluid application for non-septic patients in ICU.

Results: Twenty-two RCTs were included, involving 6,064 non-septic ICU patients. Compared with the other fluids, $6 \%$ HES was not associated with decreased overall mortality $\left(\mathrm{RR}=1.03,95 \% \mathrm{Cl}: 0.09\right.$ to $\left.1.17 ; P=0.67 ; P^{2}=0\right)$. There was no significant difference in RRT incidence, bleeding volume and red blood cell transfusion between $6 \%$ HES group and the other fluid groups. However, patients in HES group received less total intravenous fluids than those receiving crystalloids during the first day in ICU (SMD $=-0.84 ; 95 \% \mathrm{Cl}:-1.39$ to $-0.30 ; P=0.003, P=74 \%)$.

Conclusions: This meta-analysis found no increased mortality, RRT incidence, bleeding volumes or RBC transfusion in non-septic ICU patients, but the sample sizes were small and the studies generally were of poor quality.

\section{Introduction}

Since the 1970s, hydroxyethyl starch (HES) has been widely used for volume expansion therapy. A cross-sectional study reported that about $37.1 \%$ patients in the ICU received daily plasma volume expansion, mostly using HES [1]. Another study showed that rapid intravenous infusion of HES increases cardiac output and expands blood volume more effectively than crystalloids [2]. Numerous reviews without meta-analyses also support the clinical application of HES $[3,4]$.

\footnotetext{
* Correspondence: docwangyw@sina.com; pfdbsxubin@163.com ${ }^{\dagger}$ Equal contributors

'Department of Anesthesiology and SICU, Xinhua Hospital, Shanghai Jiaotong University, School of Medicine, Kongjiang Road 1665, Shanghai 200092, China

${ }^{5}$ Department of Hepato-Biliary-Pancreatic Surgery, Shanghai Tenth People's Hospital, Tongji University, School of Medicine, Yanchang Road 301, Shanghai 200072, China

Full list of author information is available at the end of the article
}

However, the safety of HES for plasma volume expansion is still under research, especially as many studies by Boldt have been retracted due to scientific misconduct. Recently, three large-sample randomized controlled trials (RCTs) [5-7] and meta-analyses [8-10] have indicated that HES increases the need for renal replacement therapy (RRT) in critically ill patients and mortality in sepsis patients. The statement issued by the European Society of Intensive Care Medicine (ESICM) recommends that products containing HES should not be used in septic patients (1B), other intensive care patients at a high risk of acute kidney injury (AKI) (1C) or patients with head injury or intracranial bleeding (1C) [11]. However, another international multi-center large-sample RCT reported the use of colloids (including HES, gelatin, dextran and albumin) versus any crystalloids for volume expansion therapy to decrease 90-day mortality without increasing the need for in ICU patients [12]. Hence, we wanted to further study the effect of the use of HES in ICU patients. 
There are also many non-septic patients in the ICU who require volume expansion therapy, such as those with acute hypovolemia arising from trauma, bleeding or surgery. So far, there is a lack of effective and comprehensive evidence-based trials focusing on the safety of HES for non-septic patients in the ICU. However, Zarychanski et al. [8] have reported on the use of HES in critically ill patients, and performed subgroup analyses to compare trials of septic versus non-septic patients in their meta-analysis, the results were inconsistent between subgroups. After exclusion of the Boldt' papers HES was shown to significantly increase the risk of mortality in all critically ill patients. However, HES did not increase the mortality in the non-septic subgroup, which was contradictory to the result obtained from the septic subgroup in their paper. Furthermore, subgroup analysis was performed only for mortality; AKI and RRT incidence were not reported in the non-septic subgroup. Mutter et al. [9] made a systematic review to assess the effects of HES versus other fluids on kidney function in all patient populations. HES products were found to increase the risk in AKI and RRT in all patients. Surprisingly, Mutter et al. detected a significant decrease in risk and injury of renal function according to the risk, injury, failure, loss of kidney function and end-stage kidney disease (RIFLE) criteria in the non-septic subgroup treated with HES versus other fluids [9].

Two meta-analyses published recently demonstrated that use of HES for volume expansion therapy during surgery was not associated with increased postoperative mortality or RRT use [13,14], which differed from the studies focusing on septic patients. However, Cittanova et al. reported that HES significantly increases serum creatinine concentrations during the first 8 days after transplantation in kidney-transplant recipients [15]. These results suggest that HES could have different effects on different diseases. Therefore, whether or not use of HES in non-septic ICU patients could induce a result similar to that seen in septic ICU patients needs to be further confirmed. The aim of the present study was to evaluate the impact of $6 \%$ HES on mortality, RRT use, bleeding volume, red blood cell (RBC) transfusion and fluid application among non-septic patients in the ICU.

\section{Materials and methods}

According to the methodology recommended by the Cochrane Collaboration [16], we included related RCTs for analyzing the safety of 6\% HES for nonseptic patients in the ICU. The primary endpoints were overall mortality and use of RRT, and the secondary endpoints were bleeding volume, RBC transfusion and fluid application. We reported the meta-analysis according to the preferred reporting items for systematic reviews and meta-analyses (PRISMA) criteria [17].

Studies were selected if they met the following criteria: 1) RCTs; 2) patient age $\geq 18$ years; 3 ) studies consisting of a group of patients in whom 6\% HES was used, and a control group receiving other intravenous fluids in the ICU; and 4) subgroups of non-septic patients who were reported to have received 6\% HES and other intravenous fluids in the ICU. Studies were excluded if they had any of the following characteristics: 1) septic patients as research subjects; 2) no group receiving 6\% HES; 3) no data available and 4) Boldt's research studies.

\section{Search strategy}

We searched Pubmed, OvidSP, Embase database and the Cochrane Library, including reference lists of relevant clinical trials, systematic reviews and meta-analyses published before November 2013, and that met the above criteria. The term in MeSH was "Hetastarch" and related free words were also searched such as "hydroxyethylstarch", "HES", "Tetraspan", "Voluven" and so on. The search was limited by "RCTs", "human" and "adult". "Language" was not a restricted searching condition. Details are provided in Additional file 1.

\section{Data extraction}

Two reviewers $(\mathrm{BH}, \mathrm{BX})$ independently screened the results of the retrieved and acquired full texts that met the above criteria. For each acquired article, the two reviewers independently extracted the valid data, including overall mortality, RRT use, bleeding volume, $\mathrm{RBC}$ transfusion, and fluid application during the first day in ICU. A third reviewer (XX) would arbitrate in the event of any disagreement between the two reviewers.

\section{Risk of bias assessment and study quality}

The Cochrane Collaboration risk-of-bias tool [16] was used to evaluate the internal validity of the included articles. The tool contained the following items: generation of random sequence, allocation concealment, blinding, incomplete data reporting, selective reporting results and other problems that could put the study at a risk of bias. Quality assessment was evaluated using mthe odified Jadad score [18]. The scale is a score from 0 to 7 (highest level of quality) according to generation of random sequence, allocation concealment, blinding, and withdrawals of clinical trials. High quality was defined by a Jadad score of 4 to 7 ; low quality was defined by a Jadad score $\leq 3$.

\section{Statistical analysis}

Review Manager (RevMan, version 5.2) was used to analyze the included studies and data. Standard mean difference (SMD) was used for pooling continuous data. When median and extreme values were presented in the 
original articles, these data were converted into mean and SD according to relevant formulas [19]. If median and quartile range were reported, mean value and SD were estimated by the method provided in the 7.7.3.5 section of the Cochrane Handbook [16]. If the HES groups or control groups included more than one group, the respective data were pooled (weighted estimate) according to the Cochrane Handbook (7.7.3.8 section) [16]. For non-continuous data, relative risk (RR) was adopted. Heterogeneity was quantified using the $I^{2}$-test [20]. The fixed effects model was selected if there was no heterogeneity $\left(I^{2}<50 \%\right)$, and the random effects model was selected in the event of $50 \% \leq I^{2}<75 \%$. A sensitivity analysis or subgroup analysis was performed to exclude the heterogeneity if $I^{2}$ was $\geq 75 \%$, otherwise meta-analysis was not carried out. Publication bias was tested using funnel plots and the Egger's test [21]. Two-sided tests were performed with a significant difference at $P<0.05$.

\section{Results}

\section{RCTs included}

The flow diagram of this meta-analysis is presented in Figure 1. A total of 2,919 articles were retrieved, of which 2,897 were excluded. Finally, 22 eligible RCTs were included in this meta-analysis according to the inclusion and exclusion criteria.
Characteristics of the included studies and study quality The characteristics of the 22 included RCTs are shown in Table 1. Of these, 14 RCTs reported patients who had undergone complex surgical procedures needing volume expansion therapy in the ICU [22-35], and 8 RCTs reported patients with other diseases (such as cardiac arrest, trauma, and so on) who received volume expansion therapy in the ICU [6,36-42]. Two RCTs reported data for a non-septic subgroup [6,38], and we included these data. These studies used different indicators to evaluate whether patients reached circulation stabilization. For example, one study used cardiac index [22], some chose urine and central venous pressure $[37,41,42]$, and others combined several indicators [23,24,28-32,39].

Among our included articles, 14 RCTs [6,23-27,32,34, 36,38-42] reported the overall mortality; 9 RCTs [24-27,31,34,37,41,42] reported the incidence of RRT; 10 RCTs [22-24,27-31,33,35] reported bleeding volume after surgery; 10 RCTs [22,24-28,30,31,33,35] reported RBC transfusion after surgery; and 9 RCTs [23,27-30, $32,33,37,42]$ reported fluid application during the first day in ICU (Additional file 2: Table S1). According to the modified Jadad score, high quality was determined in 13 studies, and low quality was determined in 9 studies (Table 2).

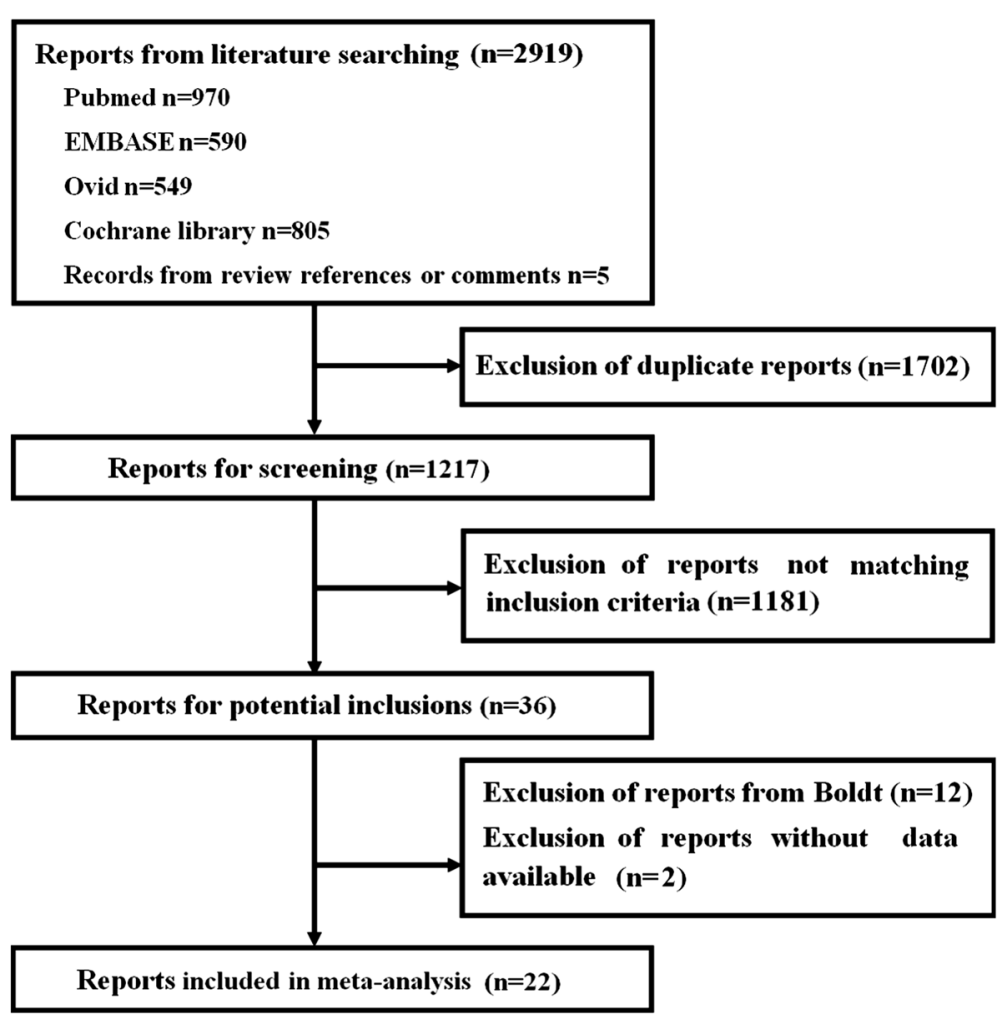

Figure 1 Flow diagram of this meta-analysis. 
Table 1 Characteristics of articles included, patient diagnosis, number of participants, interventions, and related details of reports

\begin{tabular}{|c|c|c|c|c|c|c|c|c|c|c|}
\hline \multirow{3}{*}{$\begin{array}{l}\text { Author, year } \\
\text { Ley, } 1990 \text { [22] }\end{array}$} & \multirow{3}{*}{$\begin{array}{l}\text { Diagnosis } \\
\text { After cardiac surgery }\end{array}$} & \multirow{3}{*}{$\begin{array}{l}\text { Patients } \\
\text { (n) }\end{array}$} & \multicolumn{2}{|l|}{ Intervention } & \multicolumn{2}{|l|}{ Control } & \multirow{2}{*}{$\begin{array}{l}\text { Total dose of hydroxyethyl } \\
\text { starch (HES) }\end{array}$} & \multirow{2}{*}{$\begin{array}{l}\text { Volume expansion } \\
\text { goals }\end{array}$} & \multirow{2}{*}{$\begin{array}{l}\text { Intervention } \\
\text { period }\end{array}$} & \multirow{2}{*}{$\begin{array}{l}\text { PD or } \\
\text { CA }\end{array}$} \\
\hline & & & Study fluids & n1 & Control fluids & & & & & \\
\hline & & & $6 \%$ HES 450/0.7 11 & & Normal saline & 10 & Up to $1.5 \mathrm{~L}$ & $\mathrm{Cl}>2.0 \mathrm{~L} / \mathrm{min} / \mathrm{m}^{2}$ & NS & $P D$ \\
\hline Beards,1994 [36] & Hypovolemia & 25 & 6\% HES 450/0.7 13 & & Gelatin & 12 & $500 \mathrm{~mL}$ bolus & NS & 15 to 30 minutes & PD \\
\hline \multirow[t]{3}{*}{ Van der Linden, 2005 [23] } & \multirow[t]{3}{*}{ After cardiac surgery } & \multirow[t]{3}{*}{132} & \multirow[t]{3}{*}{$6 \%$ HES 130/0.4 } & \multirow[t]{3}{*}{64} & \multirow[t]{3}{*}{ Gelatin } & \multirow[t]{3}{*}{68} & \multirow{3}{*}{$\begin{array}{l}\text { Maximum } \\
50 \mathrm{~mL} / \mathrm{kg} / \text { day }\end{array}$} & $\mathrm{PAOP}, 8$ to $15 \mathrm{mmHg}$ & \multirow[t]{3}{*}{ NS } & \multirow[t]{3}{*}{ PD } \\
\hline & & & & & & & & $\mathrm{Cl}>2.5 \mathrm{~L} / \mathrm{min} / \mathrm{m}^{2}$ & & \\
\hline & & & & & & & & Urine $>0.5 \mathrm{~mL} / \mathrm{kg} / \mathrm{h}$ & & \\
\hline \multirow[t]{2}{*}{ Chen, 2006 [37] } & \multirow[t]{2}{*}{ Burn } & \multirow[t]{2}{*}{66} & \multirow[t]{2}{*}{$6 \%$ HES 130/0.4 } & \multirow[t]{2}{*}{33} & \multirow[t]{2}{*}{ Plasma } & \multirow[t]{2}{*}{33} & \multirow[t]{2}{*}{$2328 \pm 271 \mathrm{~mL}$} & CVP 7 to $14 \mathrm{mmHg}$ & \multirow[t]{2}{*}{$48 \mathrm{~h}$} & CA \\
\hline & & & & & & & & Urine $>0.5 \mathrm{~mL} / \mathrm{kg} / \mathrm{h}$ & & \\
\hline Mahmood, 2007 [24] & After aortic aneurysm & 62 & $6 \%$ HES 130/0.4 & 21 & Gelatin & 20 & Surgery, $3 \mathrm{ml} / \mathrm{kg} / \mathrm{h}$ & MAP $>85 \mathrm{mmHg}$ & Surgery and ICU & PD \\
\hline & & & $6 \%$ HES 200/0.62 & 21 & & & $\mathrm{CU}$, as needed & CVP 8 to $10 \mathrm{mmHg}$ & & \\
\hline Godet, 2008 [25] & $\begin{array}{l}\text { After abdominal } \\
\text { aortic surgery }\end{array}$ & 65 & $6 \%$ HES 130/0.4 & 32 & Gelatin & 33 & As needed & Doctor's judgement & $\begin{array}{l}\text { Surgery and } \\
6 \mathrm{~d} \text { in ICU }\end{array}$ & PD \\
\hline Mukhtar, 2009 [26] & $\begin{array}{l}\text { After transplantation } \\
\text { surgery }\end{array}$ & 40 & $6 \%$ HES 130/0.4 & 20 & Albumin & 20 & $\begin{array}{l}\text { Maximum } \\
50 \mathrm{~mL} / \mathrm{kg} / \text { day }\end{array}$ & CVP, 5 to $7 \mathrm{mmHg}$ & $\begin{array}{l}\text { Surgery and } \\
4 \mathrm{~d} \text { in ICU }\end{array}$ & PD \\
\hline Ooi, 2009 [27] & After cardiac surgery & 90 & $6 \%$ HES 130/0.4 & 45 & Gelatin & 45 & As needed & CVP, 10 to $14 \mathrm{mmHg}$ & NS & PD \\
\hline Schramko, 2009 [28] & After cardiac surgery & 45 & $6 \%$ HES 130/0.4 & 15 & Albumin & 15 & NS & PAWP, 10 to $14 \mathrm{mmHg}$ & NS & PD \\
\hline & & & $6 \%$ HES 200/0.62 & 15 & & & & $\mathrm{Cl}>2.0 \mathrm{~L} / \mathrm{min} / \mathrm{m}^{2}$ & & \\
\hline Choi, 2010 [29] & After abdominal & 36 & $6 \%$ HES 130/0.4 & 18 & Albumin & 18 & Maximum & PAOP, 10 to $14 \mathrm{mmHg}$ & NS & PD \\
\hline & & & & & & & $20 \mathrm{~mL} /$ & $\mathrm{Cl}>2.0 \mathrm{~L} / \mathrm{min} / \mathrm{m}^{2}$ & & \\
\hline & & & & & & & & Urine $>0.5 \mathrm{~mL} / \mathrm{kg} / \mathrm{h}$ & & \\
\hline Gondos, 2010 [38] & Hypovolemia & 106 & $6 \%$ HES 130/0.4 & 26 & $\mathrm{RL}$ & 28 & $10 \mathrm{~mL} / \mathrm{kg}$ & NS & NS & PD \\
\hline & & & & & Gelatin & 25 & & & & \\
\hline & & & & & Albumin & 27 & & & & \\
\hline Heradstveit, 2010 [39] & After cardiac arrest & 19 & $6 \%$ HES 200/0.5 & 10 & RL & 9 & maximum & MAP $>60 \mathrm{mmHg}$ & Observation & PD \\
\hline & & & & & & & 500 & $\mathrm{HR}, 60$ to $100 / \mathrm{min}$ & & \\
\hline & & & & & & & & CVP, 8 to $12 \mathrm{mmHg}$ & & \\
\hline Inal, 2010 [40] & Hypovolemia & 30 & $6 \%$ HES 130/0.4 & 15 & Gelatin & 15 & $500 \mathrm{~mL}$ & NS & NS & PD \\
\hline Schramko, 2010 [30] & After cardiac surgery & 45 & $6 \%$ HES 130/0.4 & 15 & RL & 15 & $28 \mathrm{~mL} / \mathrm{kg}$ & PCWP, 10 to $15 \mathrm{mmHg}$ & 16 to $20 \mathrm{~h}$ in ICU & PD \\
\hline & & & & & Gelatin & 15 & & $\mathrm{Cl}>2.0 \mathrm{~L} / \mathrm{min} / \mathrm{m}^{2}$ & & \\
\hline Du, $2011[41]$ & Acute pancreatitis & 41 & $6 \%$ HES 130/0.4 & 20 & RL & 21 & as needed & CVP, 8 to $15 \mathrm{mmHg}$ & $8 \mathrm{~d}$ in hospital & CA \\
\hline & & & & & & & 1:3 with saline & Urine $>0.5 \mathrm{~mL} / \mathrm{kg} / \mathrm{h}$ & & \\
\hline & & & & & & & & $\mathrm{SBP}>90 \mathrm{mmHg}$ & & \\
\hline James, 2011 [42] & Trauma & 109 & $6 \%$ HES 130/0.4 & 56 & Normal saline & 53 & $500 \mathrm{~mL}$ boluses & CVP $>12 \mathrm{mmHg}$ & 15 minutes & PD \\
\hline
\end{tabular}


Table 1 Characteristics of articles included, patient diagnosis, number of participants, interventions, and related details of reports (Continued)

\begin{tabular}{|c|c|c|c|c|c|c|c|c|c|c|}
\hline & & & & & & & & Urine $>0.5 \mathrm{~mL} / \mathrm{kg} / \mathrm{h}$ & & \\
\hline \multirow[t]{3}{*}{ Lee, 2011 [31] } & After cardiac surgery & 106 & $6 \%$ HES 130/0.4 & 53 & Crystalloid & 53 & Maximum $50 \mathrm{~mL} / \mathrm{kg} / \mathrm{d}$ & $\mathrm{Cl}>2.2 \mathrm{~L} / \mathrm{min} / \mathrm{m}^{2}$ & NS & PD \\
\hline & & & & & & & & $\mathrm{SvO} 2>60 \%$ & & \\
\hline & & & & & & & & Urine $>0.5 \mathrm{~mL} / \mathrm{kg} / \mathrm{h}$ & & \\
\hline \multirow[t]{2}{*}{ Yang, 2011 [32] } & After hepatectomy & 81 & $6 \%$ HES 130/0.4 & 26 & RL & 25 & $1,000 \mathrm{~mL} / \mathrm{d}^{*} 3 \mathrm{~d}$ & CVP, 5 to $9 \mathrm{mmHg}$ & $5 d$ & CA \\
\hline & & & & & Albumin & 30 & $500 \mathrm{~mL} / \mathrm{d}^{*} 2 \mathrm{~d}$ & $\mathrm{MAP}, 60$ to $80 \mathrm{mmHg}$ & & \\
\hline Myburgh, 2012 [6] & Non-septic patients & 4720 & $6 \%$ HES 130/0.4 & 2337 & Normal saline & 2383 & $\begin{array}{l}500 \mathrm{~mL} \text { bolus, } \\
\text { maximum } 50 \mathrm{~mL} / \mathrm{kg} / \mathrm{d}\end{array}$ & NS & $90 d$ & CA \\
\hline \multirow[t]{2}{*}{ Alavi, 2012 [33] } & After cardiac surgery & 92 & $6 \%$ HES 130/0.4 & 32 & RL & 29 & As needed & CVP, 7 to $14 \mathrm{mmHg}$ & Surgery and ICU & PD \\
\hline & & & & & Gelatin & 31 & & & & \\
\hline Nagpal D, 2012 [34] & After cardiac surgery & 70 & $6 \%$ HES 130/0.4 & 35 & Crystalloid & 35 & 1 to $3.0 \mathrm{~L} / \mathrm{d}$ & NS & NS & PD \\
\hline Kimenai, 2013 [35] & After cardiac surgery & 60 & $6 \%$ HES 130/0.4 & 30 & Gelatin & 30 & NS & NS & NS & PD \\
\hline
\end{tabular}

RL, Ringer lactate; $n$, number of overall patients; $n 1$, number of patients in intervention group; $n 2$ number of patients in control group; NS, not stated; Cl, cardiac index; PAOP, pulmonary artery occlusive pressure; CVP, central venous pressure; MAP, mean arterial pressure; PAWP, pulmonary artery wedge pressure; HR, heart rate; PCWP, pulmonary capillary wedge pressure; SBP systolic blood pressure; SvO2, mixed venous oxygen saturation; PD, published data; CA, connected with author successfully. 
Table 2 Risk of bias and literature quality

\begin{tabular}{|c|c|c|c|c|c|c|c|}
\hline Trials & $\begin{array}{l}\text { Random sequence } \\
\text { generation }\end{array}$ & $\begin{array}{l}\text { Allocation } \\
\text { concealment }\end{array}$ & Blinding & $\begin{array}{l}\text { Incomplete outcome } \\
\text { data treatment }\end{array}$ & $\begin{array}{l}\text { Selective outcome } \\
\text { reporting }\end{array}$ & $\begin{array}{l}\text { Other } \\
\text { bias }\end{array}$ & $\begin{array}{l}\text { Jadad } \\
\text { score }\end{array}$ \\
\hline \multicolumn{8}{|l|}{ High Quality } \\
\hline Van der Linden, 2005 [23] & Low & Unclear & High & Low & Low & Low & 4 \\
\hline Mahmood, 2007 [24] & Low & Low & High & Low & Low & Low & 5 \\
\hline Godet, 2008 [25] & Low & Low & High & Low & Low & High & 4 \\
\hline Mukhtar, 2009 [26] & Unclear & Low & Unclear & Low & Low & Unclear & 4 \\
\hline Schramko, 2009 [28] & Unclear & Low & Unclear & Low & Low & Low & 4 \\
\hline Gondos, 2010 [38] & Unclear & Low & Unclear & Low & Low & Low & 4 \\
\hline Schramko, 2010 [30] & Unclear & Low & High & Low & Low & Low & 4 \\
\hline Du, $2011[41]$ & Low & Unclear & Unclear & High & High & Low & 4 \\
\hline James, 2011 [42] & Low & Low & Low & High & Low & Low & 6 \\
\hline Myburgh, 2012 [6] & Low & Low & Low & Low & Low & Low & 7 \\
\hline Alavi, 2012 [33] & Unclear & Low & Low & Low & Low & Low & 4 \\
\hline Nagpal, 2012 [34] & Low & Low & Low & Unclear & Low & Low & 5 \\
\hline Kimenai, 2013 [35] & Low & Unclear & High & Low & Low & Low & 4 \\
\hline \multicolumn{8}{|l|}{ Low Quality } \\
\hline Ley, 1990 [22] & Unclear & Unclear & Unclear & Low & Low & Unclear & 2 \\
\hline Berard, 1994 [36] & Unclear & Unclear & Unclear & Low & Low & Low & 2 \\
\hline Chen, 2006 [37] & Low & Unclear & High & Low & Low & Unclear & 3 \\
\hline Ooi, 2009 [27] & Unclear & Unclear & High & Low & Unclear & Low & 2 \\
\hline Choi, 2010 [29] & Low & Unclear & Unclear & Low & Low & Low & 3 \\
\hline Heradstveit, 2010 [39] & Unclear & Unclear & High & Low & Low & High & 2 \\
\hline Inal, 2010 [40] & Unclear & Unclear & Unclear & Low & Unclear & Low & 2 \\
\hline Lee, 2011 [31] & Unclear & Unclear & High & Low & Low & Low & 2 \\
\hline Yang, 2011 [32] & Low & Unclear & Unclear & High & Low & Low & 3 \\
\hline
\end{tabular}

\section{Overall mortality}

A total of 14 articles reported the overall mortality, involving 5,593 patients. Compared with the other types of fluids (crystalloids, gelatine or albumin), the use of $6 \%$ HES was not associated with decreased overall mortality $\left(\mathrm{RR}=1.03,95 \%\right.$ CI 0.90 to $\left.1.17 ; \quad P=0.67 ; \quad I^{2}=0\right)$
(Figure 2). Publication bias was not found by the Egger's test $(P=0.85)$ or funnel plots (Figure 3$)$.

\section{Renal replacement therapy}

Nine articles reported the incidence of RRT, showing that $6 \%$ HES did not increase RRT use as compared with

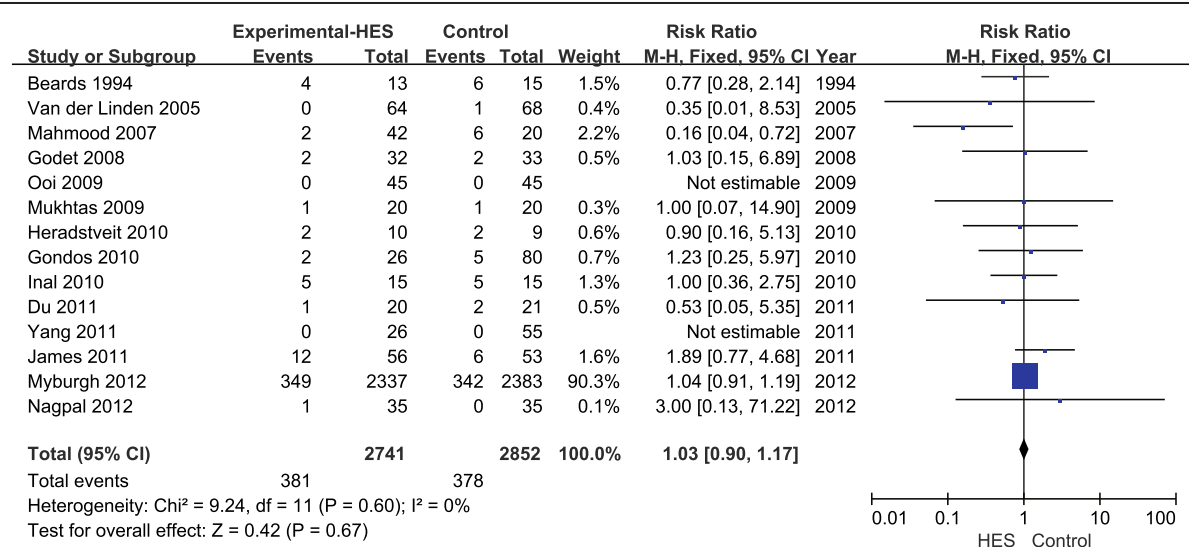

Figure 2 Forest plot of pooled risk ratio for overall mortality. HES, hydroxethyl starch. M-H, Mantel-Haenszel. 


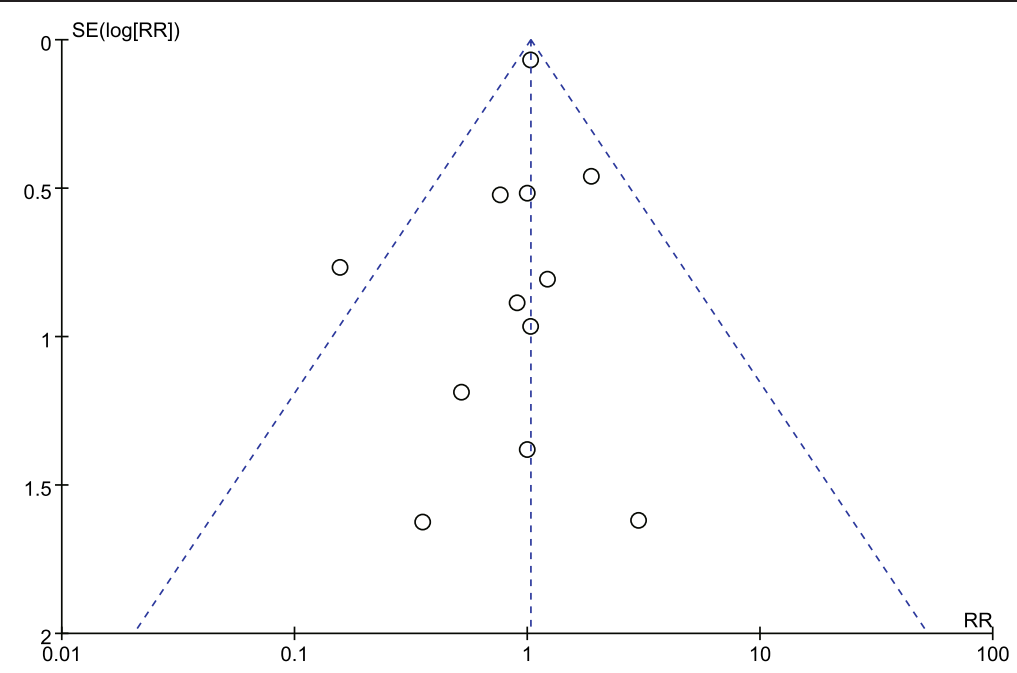

Figure 3 Funnel plot of overall mortality. RR, relative risk. SE, standard error.

the other fluids ( $\mathrm{RR}=0.83 ; 95 \% \mathrm{CI} 0.36$ to $1.91 ; P=0.67$; $I^{2}=0 \%$ ) (Figure 4). Funnel plots showed no publication bias (Figure 5), and the $P$-value from Egger's test was 0.58 .

\section{Bleeding volume and RBC transfusion}

Bleeding volume was reported in 10 articles. Of these, we only pooled seven articles [22,27,28,30,31,33,35] in this meta-analysis, which all reported bleeding volume after surgery. Three articles [23,24,29] were excluded, as bleeding volume was reported from surgery or during several periods after surgery. Data from Schramko et al., Alavi et al. and Kimenai et al. were converted into mean and SD according to the method above $[16,19]$. There was no significant difference in bleeding volume between the $6 \%$ HES group and other fluid groups $(\mathrm{SMD}=-0.10$, 95\% CI -0.29 to $0.08 ; P=0.28 ; I^{2}=0 \%$ ) (Figure 6A). Egger's test showed no publication bias $(P=0.35)$.

Nine articles reported RBC transfusion, and five articles [22,23,26,31,42] reporting $\mathrm{RBC}$ transfusion after surgery were included and showed no significant difference between the HES group and other fluids group
$\left(\mathrm{SMD}=-0.13 ; 95 \% \mathrm{CI}-0.33\right.$ to $\left.0.08 ; P=0.23 ; I^{2}=4 \%\right)$ (Figure 6B). Egger's test showed no publication bias $(P=0.51)$. Four articles were excluded because two of them $[28,33]$ only reported overall volume of $\mathrm{RBC}$ transfusion, and the other two [24,26] reported $\mathrm{RBC}$ transfusion before surgery. Data from three articles were transformed $[23,26,42]$.

\section{Fluid application}

Fluid application during the first day in ICU was reported in nine articles, but there was significant data heterogeneity $\left(I^{2}=94 \%\right)$. Knowing that colloids and crystalloids have different effects on volume expansion, a subgroup analysis was performed by the type of fluid used. Patients receiving $6 \%$ HES needed fewer total intravenous fluids than those receiving crystalloids (SMD $=-0.84,95 \% \mathrm{CI}-1.39$ to -0.30 ; $P=0.003 ; I^{2}=74 \%$ ) (Figure $7 \mathrm{~A}$ ). There was no significant difference between the 6\% HES group and albumin group $\left(\mathrm{SMD}=0.26,95 \% \mathrm{CI}-0.17\right.$ to $\left.0.70 ; P=0.23 ; I^{2}=13 \%\right)$ (Figure 7C). In this subgroup, data from Choi et al. [29] were excluded, because they were reported in several



Figure 4 Forest plot of pooled risk ratio for use of renal replacement therapy. HES, hydroxethyl starch. M-H, Mantel-Haenszel. 


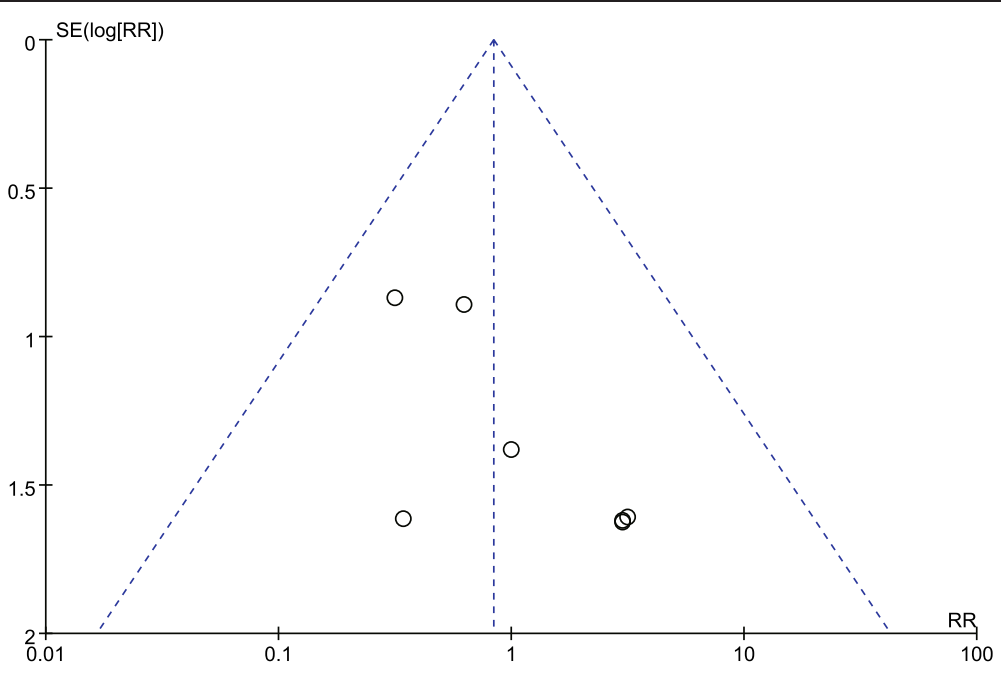

Figure 5 Funnel plot of incidence for renal replacement therapy. RR, relative risk. SE, standard error.

periods during the first day in ICU, and we failed to obtain the data from the authors. Although subgroup analysis was carried out, there remained great heterogeneity in the fluid application between the HES group and the gelatin group. A sensitivity analysis identified that the trial from Alavi et al. [33] was responsible for the heterogeneity. When their data were excluded, there was no significant difference in fluid application between the HES group and the gelatin group $(\mathrm{SMD}=-0.12,95 \% \mathrm{CI}-0.37$ to 0.13 ; $\left.P=0.35 ; I^{2}=0 \%\right)$ (Figure 7B).

\section{Length of ICU and hospital stay}

Eleven articles reported the length of ICU stay, and nine articles reported the length of hospital stay. However, significant heterogeneity was detected in both (ICU stay:
$I^{2}=85 \%$; hospital stay: $I^{2}=78 \%$ ). As the heterogeneity could not be excluded through subgroup analysis or sensitivity analysis, a description was provided instead of performing a meta-analysis. These data are not shown.

\section{Discussion}

The main finding of this meta-analysis showed that HES was not associated with decreased overall mortality and RRT use. These findings were similar with subgroup analyses of non-septic patients among critically ill patients or among all patients $[8,9]$. Our results were somewhat different from a previous meta-analysis of fluid therapy in critically ill patients, especially septic patients [43]. One major cause was that the existing data for these patients treated with HES was poor and scarce, which meant the

\section{A

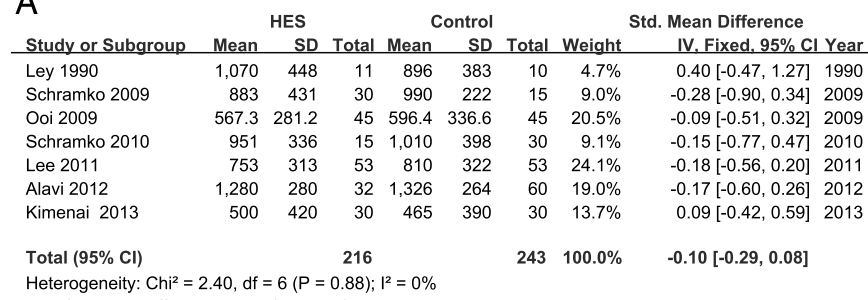 Test for overall effect: $Z=1.08(P=0.28)$}

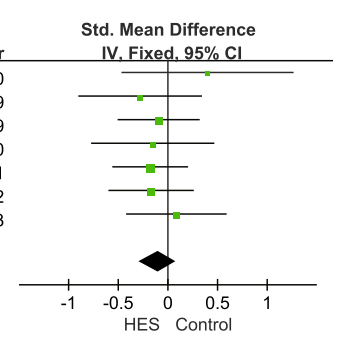

\section{B}

\begin{tabular}{|c|c|c|c|c|c|c|c|}
\hline \multirow{2}{*}{ Study or Subgroup } & \multicolumn{2}{|c|}{ HES } & \multicolumn{5}{|c|}{ Control } \\
\hline & Mean & SD & Total & Mean & SD & Tota & We \\
\hline Ley 1990 & 0.9 & 1 & 11 & 0.4 & 0.8 & 10 & \\
\hline Van der Linden 2005 & 1.5 & 1.8 & 64 & 1.5 & 1.8 & 68 & \\
\hline Mukhtas 2009 & 3 & 2.4 & 20 & 4 & 2.3 & 20 & \\
\hline James 2011 & 5.2 & 5.2 & 36 & 6 & 4.5 & 31 & \\
\hline Lee 2011 & 0.6 & 1.1 & 53 & 0.9 & 1 & 53 & \\
\hline Total $(95 \% \mathrm{Cl})$ & & & 184 & & & 182 & \\
\hline $\begin{array}{l}\text { Heterogeneity: } \\
\text { Test for overall }\end{array}$ & $\begin{array}{l}\mathrm{df}= \\
1.21\end{array}$ & & $\begin{array}{l}0.38 \\
3)\end{array}$ & & & & \\
\hline
\end{tabular}

Std. Mean Difference IV. Fixed. 95\% Cl Year $0.53[-0.35,1.40] 1990$ $0.00[-0.34,0.34] 2005$ $-0.42[-1.04,0.21] 2009$ $-0.16[-0.64,0.32] 2011$ $-0.28[-0.67,0.10] 2011$

$-0.13[-0.33,0.08]$

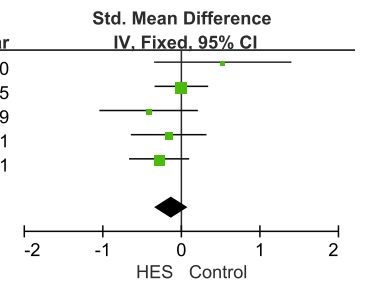

Figure 6 Forest plots of pooled estimates for bleeding volume and red blood cell transfusion. (A) Bleeding volume after surgery. (B) Red blood cell transfusion (unit). HES, hydroxethyl starch. IV, Inverse Variance. 


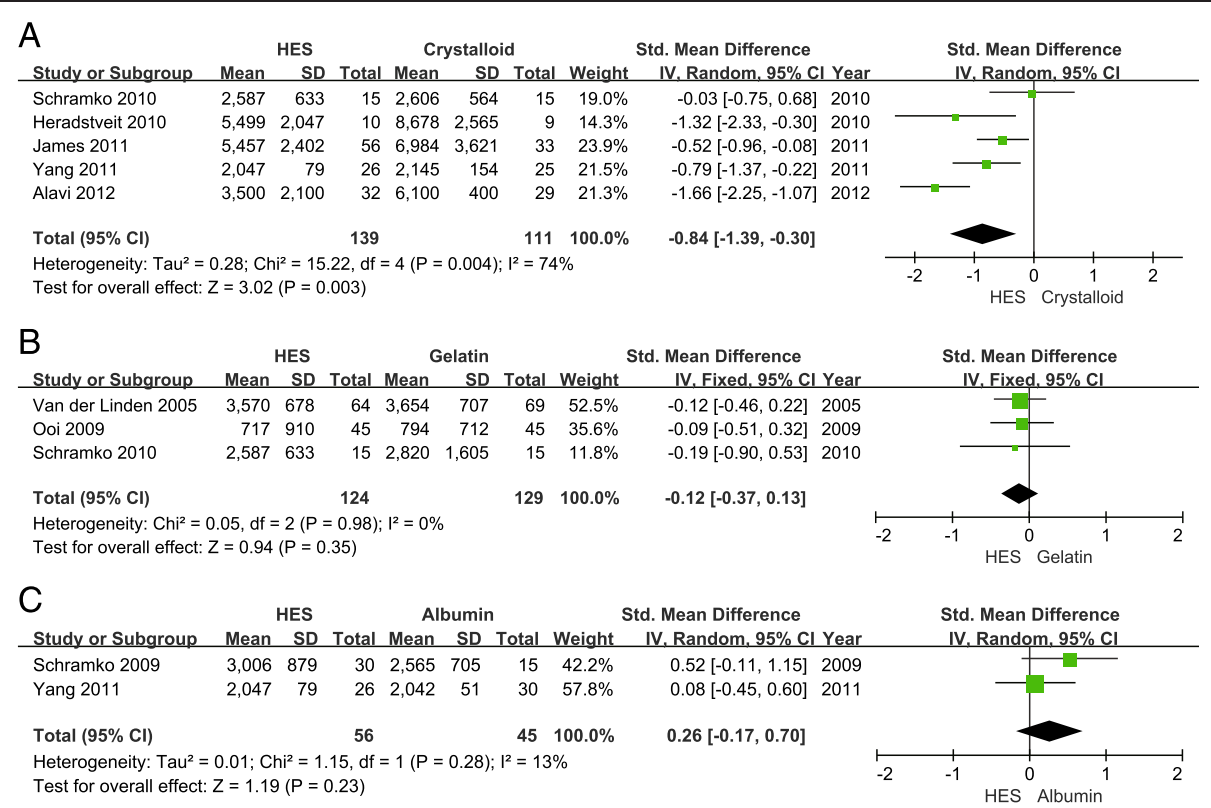

Figure 7 Forest plots of pooled estimates for fluid application $(\mathrm{mL})$ during the first day in the ICU. (A) Hydroxethyl starch (HES) versus crystalloid. (B) HES versus gelatin. (C) HES versus albumin. IV, Inverse Variance.

results needed to be verified with more data of high quality. Another reason might also be that the pathophysiological changes in these non-septic patients are different from those in septic patients, and sepsis itself could significantly increase the risk of AKI [44]. Micro leakage of capillary blood vessels was a key pathophysiologic mechanism in sepsis [45], and endothelial glycocalix plays an important role in micro leakage of capillary blood vessels [46]. Steppan et al. found that significantly more flaking of the endothelial glycocalix occurred in patients with sepsis than in patients who underwent major abdominal surgery [47]. In addition, sepsis could activate the renin-angiotensin-aldosterone system (RAAS) in renal sympathetic and angiotensin activities, which leads to vasoconstriction in patients with sodium and water retention. As a result, septic patients are predisposed to acute renal failure [44]. Hence, the side effects of HES might be more serious for septic patients than non-septic patients in the ICU.

Although several studies tried to analyze the effect of HES in a non-septic subgroup, the results of their subgroup analyses were always different from their main results $[8,9]$. In addition, their analyses were always part of the side effects of HES. Hence, we have made a meta-analysis to study the effect HES versus other fluids for non-septic ICU patients, including mortality, RRT use, bleeding volume, RBC transfusion and fluid application. This analysis was relatively comprehensive and with less heterogenicity in non-septic patients. Furthermore, it reminded us to pay more attention to volume expansion therapy in ICU non-septic patients.
The pooled analysis of overall mortality did not display more harm with HES, which was not entirely the same as previous trials [5-7] and meta-analyses $[8,10,48,49]$. Knowing that the pooled analysis of mortality may be influenced by study quality and the follow-up period, it is difficult to explain this result. On the one hand, HES may have different effects in different diseases $[5-7,13,14]$. On the other hand, study quality and the follow-up period may induce bias in our meta-analysis, as most included RCTs were small-sample studies and with short follow-up periods. Hence, we should be cautious when selecting fluids for ICU non-septic patients. In addition, the article from CHEST [6] alone accounted for $88.9 \%$ of the weighting, and the results, which played an important role in our meta-analysis, might only be suitable for the specific clinical conditions in that study (for example, a small dose of HES was chosen). We have performed a sensitivity analysis, and its exclusion did not influence the significance of the effect on overall mortality $\left(\mathrm{RR}=0.83,95 \%\right.$ CI 0.56 to $1.24 ; P=0.37 ; I^{2}=0$ ). However, other included studies were all with very small-scale samples; more high-quality RCTs focusing on 6\% HES in non-septic ICU patients are needed to confirm our results.

Efforts were also made to determine the effect of $6 \%$ HES on renal function. However different indicators were used to test renal function, such as blood creatinine [32], glomerular filtration rate [30], the incidence of AKI based on different criteria [24,34,42] and RRT use [24-27,31,34,37,41,42]. The result need further confirmation because of the limitations of our included RCTs. 
There was no significant difference in bleeding volume and RBC transfusion between the 6\% HES group and the other fluid groups. Patients in the HES group received fewer total intravenous fluids than those receiving crystalloids during the first day in ICU, which might mean that $6 \%$ HES had a better volume-expansion effect than crystalloids. However, due to the absence of demonstrable benefit, more large-scale RCTs are needed to confirm these results.

The implementation of our meta-analysis is in accordance with the requirements of the Cochrane Collaboration. These requirements include a literature search without language limitations, strict inclusion and exclusion criteria, selection of articles and collection of data by two independent authors, and bias risk evaluation. Indeed, there are several limitations in our meta-analysis. Despite widespread use of HES for more than three decades, RCTs comparing HES with other fluids for ICU non-septic patients are few, with small sample sizes, and vulnerable to bias. In addition, several control groups in the included studies used gelatin, which clouds or conceals the adverse effects of HES to some extent. Bleeding volume analysis is limited by clinical heterogeneity across studies because patients have undergone different types of surgery.

\section{Conclusion}

Although volume expansion with 6\% HES did not seem to increase the mortality or RRT use in non-septic ICU patients, the sample sizes in our meta-analysis were small and the studies generally were of poor quality.

\section{Key messages}

- The safety of HES for non-septic patients in the ICU remains elusive

- Available data from systematic reviews and meta-analyses displayed inconsistent results between septic and non-septic patients using HES

- Use of $6 \%$ HES did not seem to increase the mortality or RRT incidence in non-septic ICU patients

\section{Additional files}

Additional file 1: A priori design, electronic search strategy and studies excluded from this review.

Additional file 2: Table S1. Observation period for outcomes.

\section{Abbreviations}

AKI: acute kidney injury; ESICM: European Society of Intensive Care Medicine; HES: hydroxyethyl starch; RAAS: renin-angiotensin-aldosterone system; RBC: red blood cells; RCT: randomized controlled trial; RIFLE criteria: risk injury, failure, loss of kidney function and end-stage kidney disease; RR: relative risk; RRT: renal replacement therapy; SMD: standard mean difference; RBC: red blood cell.

\section{Competing interests}

The authors declare that they have no competing interests.

\section{Authors' contributions}

$\mathrm{BH}$ participated in data selection, data analysis and the manuscript drafting. BX (Bo Xu) also participated in data selection, data analysis and the manuscript drafting. XX helped to choose RCTs and analyze data. LL carried out the assessment of study quality and checked the data. RR participated in the design of the study and performed part of the statistical analysis. ZC helped to carry out the statistical analysis and revise the manuscript. JX performed part of the statistical analysis. BX (Bin Xu) participated in the design of the study and the revision of manuscript. YW conceived of the study and helped to revise the manuscript. All authors read and approved the manuscript.

\section{Acknowledgements}

The work was supported by the National Natural Science Foundation of China (81100826, 81470390, 81001007, 81000787, and 81200181).

\section{Author details}

${ }^{1}$ Department of Anesthesiology and SICU, Xinhua Hospital, Shanghai Jiaotong University, School of Medicine, Kongjiang Road 1665, Shanghai 200092, China. ²Department of Epidemiology, Shanghai Jiaotong University, School of Medicine, Kongjiang Road 1665, Shanghai 200092, China.

${ }^{3}$ Pharmaceutical Department, Xinhua Hospital, Shanghai Jiaotong University, School of Medicine, Kongjiang Road 1665, Shanghai 200092, China. ${ }^{4}$ Department of Hepato-Biliary-Pancreatic Surgery, Second Military Medical University, Fengyang Road 415, Shanghai 200003, China. ${ }^{5}$ Department of Hepato-Biliary-Pancreatic Surgery, Shanghai Tenth People's Hospital, Tongji University, School of Medicine, Yanchang Road 301, Shanghai 200072, China.

Received: 6 July 2014 Accepted: 20 February 2015

Published online: 19 March 2015

\section{References}

1. Finfer S, Liu B, Taylor C, Bellomo R, Billot L, Cook D, et al. Resuscitation fluid use in critically ill adults: an international cross-sectional study in 391 intensive care units. Crit Care. 2010;14:R185.

2. Mcllroy DR, Kharasch ED. Acute intravascular volume expansion with rapidly administered crystalloid or colloid in the setting of moderate hypovolemia. Anesth Analg. 2003;96:1572-7.

3. Westphal M, James MF, Kozek-Langenecker S, Stocker R, Guidet B, Van Aken H. Hydroxyethyl starches: different products-different effects. Anesthesiology. 2009;111:187-202.

4. Niemi TT, Miyashita R, Yamakage M. Colloid solutions: a clinical update. J Anesth. 2010;24:913-25.

5. Brunkhorst FM, Engel C, Bloos F, Meier-Hellmann A, Ragaller M, Weiler N, et al. Intensive insulin therapy and pentastarch resuscitation in severe sepsis. N Engl J Med. 2008;358:125-39.

6. Myburgh JA, Finfer S, Bellomo R, Billot L, Cass A, Gattas D, et al. Hydroxyethyl starch or saline for fluid resuscitation in intensive care. N Engl J Med. 2012;367:1901-11.

7. Perner A, Haase N, Guttormsen AB, Tenhunen J, Klemenzson G, Aneman A et al. Hydroxyethyl starch 130/0.42 versus Ringer's acetate in severe sepsis. N Engl J Med. 2012;367:124-34

8. Zarychanski R, Abou-Setta AM, Turgeon AF, Houston BL, McIntyre L, Marshall JC, et al. Association of hydroxyethyl starch administration with mortality and acute kidney injury in critically ill patients requiring volume resuscitation: a systematic review and meta-analysis. JAMA. 2013;309:678-88.

9. Mutter TC, Ruth CA, Dart AB. Hydroxyethyl starch (HES) versus other fluid therapies: effects on kidney function. Cochrane Database Syst Rev. 2013;7:CD007594.

10. Gattas DJ, Dan A, Myburgh J, Billot L, Lo S, Finfer S. Fluid resuscitation with 6\% hydroxyethyl starch (130/0.4 and 130/0.42) in acutely ill patients: systematic review of effects on mortality and treatment with renal replacement therapy. Intensive Care Med. 2013;39:558-68.

11. Reinhart $K$, Perner A, Sprung CL, Jaeschke R, Schortgen F, Johan Groeneveld $A B$, et al. Consensus statement of the ESICM task force on colloid volume therapy in critically ill patients. Intensive Care Med. 2012;38:368-83.

12. Annane $D$, Siami $S$, Jaber $S$, Martin $C$, Elatrous $S$, Declère $A D$, et al. Effects of fluid resuscitation with colloids vs crystalloids on mortality in critically ill 
patients presenting with hypovolemic shock: the CRISTAL randomized trial. JAMA. 2013;310:1809-17.

13. Martin C, Jacob M, Vicaut E, Guidet B, Van Aken H, Kurz A. Effect of waxy maize-derived hydroxyethyl starch 130/0.4 on renal function in surgical patients. Anesthesiology. 2013;118:387-94.

14. Gillies MA, Habicher M, Jhanji S, Sander M, Mythen M, Hamilton M, et al. Incidence of postoperative death and acute kidney injury associated with i.v. 6\% hydroxyethyl starch use: systematic review and meta-analysis. $\mathrm{Br} J$ Anaesth. 2014;112:25-34.

15. Cittanova ML, Leblanc I, Legendre C, Mouquet C, Riou B, Coriat P. Effect of hydroxyethylstarch in brain-dead kidney donors on renal function in kidney-transplant recipients. Lancet. 1996;348:1620-2.

16. Julian PT Higgins SG. Cochrane Handbook for Systematic Reviews of Interventions Version 5.1.0. 2011: Available at:http://handbook.cochrane.org

17. Moher D, Liberati A, Tetzlaff J, Altman DG, Group P. Preferred reporting items for systematic reviews andmeta-analyses: the PRISMA statement. Ann Intern Med. 2009;151:264-9.

18. Bañares R, Albillos A, Rincón D, Alonso S, González M, Ruiz-del-Arbol L, et al. Endoscopic treatment versus endoscopic plus pharmacologic treatment for acute variceal bleeding: a meta-analysis. Hepatology. 2002;35:609-15.

19. Hozo SP, Djulbegovic B, Hozo I. Estimating the mean and variance from the median, range, and the size of a sample. BMC Med Res Methodol. 2005;5:13.

20. Higgins JP, Thompson SG. Quantifying heterogeneity in a meta-analysis. Stat Med. 2002;21:1539-58.

21. Egger M, Davey Smith G, Schneider M, Minder C. Bias in meta-analysis detected by a simple, graphical test. BMJ. 1997;315:629-34.

22. Ley SJ, Miller K, Skov P, Preisig P. Crystalloid versus colloid fluid therapy after cardiac surgery. Heart Lung. 1990;19:31-40.

23. Van der Linden PJ, De Hert SG, Deraedt D, Cromheecke S, De Decker K, De Paep R, et al. Hydroxyethyl starch 130/0.4 versus modified fluid gelatin for volume expansion in cardiac surgery patients: the effects on perioperative bleeding and transfusion needs. Anesth Analg. 2005;101:629-34.

24. Mahmood A, Gosling P, Vohra RK. Randomized clinical trial comparing the effects on renal function of hydroxyethyl starch or gelatine during aortic aneurysm surgery. Br J Surg. 2007;94:427-33.

25. Godet G, Lehot JJ, Janvier G, Steib A, De Castro V, Coriat P. Safety of HES 130/0.4 (Voluven(R)) in patients with preoperative renal dysfunction undergoing abdominal aortic surgery: a prospective, randomized, controlled, parallel-group multicentre trial. Eur J Anaesthesiol. 2008;25:986-94.

26. Mukhtar A, Aboulfetouh F, Obayah G, Salah M, Emam M, Khater Y, et al. The safety of modern hydroxyethyl starch in living donor liver transplantation: a comparison with human albumin. Anesth Analg. 2009;109:924-30.

27. Ooi JS, Ramzisham AR, Zamrin MD. Is 6\% hydroxyethyl starch 130/0.4 safe in coronary artery bypass graft surgery? Asian Cardiovasc Thoracic Ann. 2009;17:368-72.

28. Schramko AA, Suojaranta-Ylinen RT, Kuitunen AH, Kukkonen SI, Niemi TT. Rapidly degradable hydroxyethyl starch solutions impair blood coagulation after cardiac surgery: a prospective randomized trial. Anesth Analg. 2009;108:30-6

29. Choi YS, Shim JK, Hong SW, Kim JC, Kwak YL. Comparing the effects of 5\% albumin and 6\% hydroxyethyl starch 130/0.4 on coagulation and inflammatory response when used as priming solutions for cardiopulmonary bypass. Minerva Anestesiol. 2010;76:584-91.

30. Schramko A, Suojaranta-Ylinen R, Kuitunen A, Raivio P, Kukkonen S, Niemi T. Hydroxyethylstarch and gelatin solutions impair blood coagulation after cardiac surgery: A prospective randomized trial. Br J Anaesth. 2010;104:691-7.

31. Lee JS, Ahn SW, Song JW, Shim JK, Yoo KJ. lan Kwak Y. Effect of hydroxyethyl starch 130/0.4 on blood loss and coagulation in patients with recent exposure to dual antiplatelet Therapy undergoing off-pump coronary artery bypass graft surgery. Circ J. 2011;75:2397-402.

32. Yang J, Wang WT, Yan LN, Xu MQ, Yang JY. Alternatives to albumin administration in hepatocellular carcinoma patients undergoing hepatectomy: An open, randomized clinical trial of efficacy and safety. Chin Med J. 2011:124:1458-64.

33. Alavi SM, Baharvand Ahmadi B, Baharestani B, Babaei T. Comparison of the effects of gelatin, Ringer's solution and a modern hydroxyl ethyl starch solution after coronary artery bypass graft surgery. Cardiovasc J Afr. 2012;23:428-31.

34. Nagpal D, NCT00964015. Starch or Saline After Cardiac Surgery (SSACS) trial. Personal Communication. 2012
35. Kimenai DM, Bastianen G, Daane CR, Megens-Bastiaanse CM, Van Der Meer NJM, Scohy TV, et al. Effect of colloids Gelatin and HES 130/0.4 on blood coagulation in cardiac surgery patients: A randomised controlled trial. Perfusion. 2013;17:140-1.

36. Beards SC, Watt T, Edwards JD, Nightingale P, Farragher EB. Comparison of the hemodynamic and oxygen transport responses to modified fluid gelatin and hetastarch in critically ill patients: a prospective, randomized trial. Crit Care Med. 1994:22:600-5.

37. Chen J, Han CM, Xia SC, Tang ZJ, Su SJ. Evaluation of effectiveness and safety of a new hydroxyethyl starch used in resuscitation of burn shock. Zhonghua Shao Shang Za Zhi. 2006;22:333-6.

38. Gondos T, Marjanek Z, Ulakcsai Z, Szabo Z, Bogar L, Karolyi M, et al. Short-term effectiveness of different volume replacement therapies in postoperative hypovolaemic patients. Eur J Anaesthesiol. 2010;27:794-800.

39. Heradstveit BE, Guttormsen AB, Langorgen J, Hammersborg SM Wentzel-Larsen T, Fanebust R, et al. Capillary leakage in post-cardiac arrest survivors during therapeutic hypothermia - a prospective, randomised study. Scand J Trauma Resusc Emerg Med. 2010;18:29.

40. Inal MT, Memis D, Karamanlioglu B, Sut N. Effects of polygeline and hydroxyethyl starch solutions on liver functions assessed with LIMON in hypovolemic patients. J Crit Care. 2010;25:361. e1-5.

41. Du XJ, Hu WM, Xia Q, Huang ZW, Chen GY, Jin XD, et al. Hydroxyethyl starch resuscitation reduces the risk of intra-abdominal hypertension in severe acute pancreatitis. Pancreas. 2011;40:1220-5.

42. James MFM, Michell WL, Joubert IA, Nicol AJ, Navsaria PH, Gillespie RS. Resuscitation with hydroxyethyl starch improves renal function and lactate clearance in penetrating trauma in a randomized controlled study: The FIRST trial (Fluids in Resuscitation of Severe Trauma). Br J Anaesth. 2011;107:693-702.

43. Haase N, Perner A, Hennings LI, Siegemund M, Lauridsen B, Wetterslev M, et al. Hydroxyethyl starch 130/0.38-0.45 versus crystalloid or albumin in patients with sepsis: systematic review with meta-analysis and trial sequential analysis. BMJ. 2013;346:f839.

44. Schrier RW, Wang W. Acute renal failure and sepsis. N Engl J Med. 2004:351:159-69.

45. Van den Berg BM, Vink H, Spaan JA. The endothelial glycocalyx protects against myocardial edema. Circ Res. 2003;92:592.

46. Marechal X, Favory R, Joulin O, Montaigne D, Hassoun S, Decoster B, et al. Endothelial Glycocalix damage during endotoxemia coincides with microcirculatory dysfunction and vascular oxidative stress. Shock. 2008;29:572

47. Steppan J, Hofer S, Funke B, Brenner T, Henrich M, Martin E, et al. Sepsis and major abdominal surgery lead to flaking of the endothelial glycocalix. J Surg Res. 2011:165:136-41.

48. Patel A, Waheed U, Brett SJ. Randomised trials of $6 \%$ tetrastarch (hydroxyethyl starch 130/0.4 or 0.42) for severe sepsis reporting mortality: systematic review and meta-analysis. Intensive Care Med. 2013;39:811-22.

49. Perel P, Roberts I, Ker K. Colloids versus crystalloids for fluid resuscitation in critically ill patients. Cochrane Database Syst Rev. 2013;2:CD000567.

\section{Submit your next manuscript to BioMed Central and take full advantage of:}

- Convenient online submission

- Thorough peer review

- No space constraints or color figure charges

- Immediate publication on acceptance

- Inclusion in PubMed, CAS, Scopus and Google Scholar

- Research which is freely available for redistribution 